\title{
Fetal Prognostics in Relation to Uricemia and Maternal Proteinuria of Arterial High Blood Pressure Types during Pregnancy at the Maternity of Donka, National Hospital Donka, CHU of Conakry, Guinea
}

\author{
Boubacar Siddi Diallo1*, Mamadou Sambakeita1, Ibrahima Sory Balde², Oumar Diawara1, \\ Telly Sy², Mohamed Lamine Kaba1, Yolande Hyjazi'1, Namory Keita1 \\ ${ }^{1}$ University Department of Gynecology-Obstetrics, Donka National Hospital, Conakry, Guinea \\ ${ }^{2}$ University Department of Gynecology-Obstetrics, Ignace Deen National Hospital, Conakry, Guinea \\ Email: *tounty1@yahoo.fr, keitamamadousamba@yahoo.fr, baldeisory@yahoo.fr, oumardiawara111@gmail.com, \\ sytelly@yahoo.fr, kabalamin@yahoo.fr,yhyjazi@jhpiego.net, namoryk2010@yahoo.fr
}

How to cite this paper: Diallo, B.S., Sambakeita, M., Balde, I.S., Diawara, O., Sy, T., Kaba, M.L., Hyjazi, Y. and Keita, N. (2019) Fetal Prognostics in Relation to Uricemia and Maternal Proteinuria of Arterial High Blood Pressure Types during Pregnancy at the Maternity of Donka, National Hospital Donka, CHU of Conakry, Guinea. Open Journal of Obstetrics and Gynecology, 9, 960-967.

https://doi.org/10.4236/ojog.2019.97093

Received: April 19, 2019

Accepted: July 8, 2019

Published: July 11, 2019

Copyright $\odot 2019$ by author(s) and Scientific Research Publishing Inc. This work is licensed under the Creative Commons Attribution International License (CC BY 4.0).

http://creativecommons.org/licenses/by/4.0/ CC) (i) Open Access

\begin{abstract}
Objectives: The objectives of this work were to calculate the frequency of arterial hypertension during pregnancy, describe the epidemiological profile, and identify the most common type of hypertension and to establish fetal prognosis based on uricemia and maternal proteinuria. Methodology: This was a six (6) month descriptive prospective study performed in the Obstetrics and Gynecology Department of Donka National Hospital-CHU Conakry. The study took place from july 1 st to December 31st, 2015. Results: The frequency of arterial hypertension during pregnancy was $8.82 \%$ in the service. The epidemiological profile was that of teenagers (32.8\%), nulliparous (56\%), coming from home (69.2\%), not having performed CPN (52\%), not schooled $(68 \%)$ and housewives. The primary factor was the risk factor (52.4\%). Gestational age greater than 37 was the most concerned (62\%). The reasons for consultation are dominated by headache (76\%) and vertigo (68\%). The main type of hypertension was pre-eclampsia (48\%) followed by Transient HTA (28\%). The predominant clinical form during the admission was pre-eclampsia (47.2\%) followed by eclampsia (23\%). At the first minute, $35.68 \%$ of newborns had an APGAR score of less than 7 and the fifth $25.5 \%$ had a score of less than 7. Fetal morbidity was dominated by fetal hypotrophy (30.19\%), followed by prematurity (23.92\%). In $90.90 \%$ of hypotrophy, there are $85.24 \%$ of premature babies, $95.55 \%$ of SFA, and $80 \%$ of MIU; the serum uric acid was greater than $350 \mathrm{mmol}$. We recorded 204 children born with moth-
\end{abstract}


ers with proteinuria greater than or equal to $30 \mathrm{mg} / \mathrm{dl}$, or $80 \%$ of children. 30 cases of MFIU and 7 cases of neonatal death out of 255 births, that is $14.50 \%$ were noted. Conclusion: The detection of risk factors by a good prenatal follow-up and the regular training of the care providers for adequate and multidisciplinary care (obstetrician, intensive care nephrologist and pediatrician) of hypertensive pregnant women and their newborns can improve the maternal prognosis and fetal.

\section{Keywords}

Uricemia, Maternal Proteinuria, Arterial High Blood Pressure

\section{Introduction}

Hypertension in pregnancy is defined as systolic blood pressure greater than or equal to $140 \mathrm{~mm} / \mathrm{Hg}$ and/or diastolic blood pressure greater than or equal to 90 $\mathrm{mm} / \mathrm{Hg}$ in two (2) consecutive visits separated by at least 4 hours in a woman resting for 10 to 15 minutes in a sitting position then in left lateral decubitus [1].

The WHO estimates that 150,000 pregnant women die each year worldwide as a result of the complications of pregnancy-induced hypertension, most of them following an eclampsia crisis [2].

The frequency of arterial hypertension during pregnancy varies from country to country; according to WHO this frequency varies between $0.1 \%$ and $31.4 \%$ of pregnancies [2]. In developed countries; it is in the order of $9 \%$ to $15 \%$ of pregnancies: in France 10\% [3]; in the United Kingdom 10.8\% [4]; in the United States $10 \%$ - 15\% [5]; in China 9, 4\% [6]. In sub-Saharan Africa we only have hospital statistics: in Madagascar 11\% [6]; in Morocco 7, 94\% [7]; in Niger 7\% [8]; in Senegal 3, 9\% [9] and Côte d'Ivoire 4, 6\% [10].

In Guinea, this frequency varies from one hospital structure to another.

Sakouvogui B found $10.35 \%$ at Kindia Regional Hospital [11] and Kougang N.E. at Ignace Deen National Hospital found 8.97\% [12].

The objectives of this work were to calculate the frequency of arterial hypertension during pregnancy, describe the epidemiological profile, identify the most common type of hypertension and establish the maternal and fetal prognosis [13].

\section{Methodology}

This was a prospective of six (6) month descriptive study performed in the Obstetrics and Gynecology Department of Donka National Hospital, CHU Conakry. The study took place from July 1st to December 31st, 2015.

$\checkmark$ It was included in the study all pregnant, parturient and postpartum women who were admitted to the department and had a blood pressure (BP) greater than or equal to $140 / 90 \mathrm{mmHg}$ during the study period.

$\checkmark$ It was not included in the study, all pregnant; parturients and women fol- 
lowing diapers admitted to the department with blood pressure (BP) less than or equal to $139 / 89 \mathrm{~mm} / \mathrm{Hg}$ during the study period (without any antihypertensive treatment) and post-hypertensive women partum.

$\checkmark$ It was excluded from the study all hypertensive patients who were not hospitalized during the study period and patients not concerned by follow-up during our study period.

The variables studied were:

$\checkmark$ Some quantitative: frequency, maternal age, parity, prenatal consultationgestational age and APGAR score.

- The other qualitative: admission mode, level of education, socio-professional layers, type of high blood pressure, period of discovery reasons for consultation, risk factors for eclampsia, clinical form at admission, maternal complications and fetal complications.

\section{Results}

1) Frequency: During our study period, we recorded 250 hypertensive women out of a total of 2833 women who gave birth in the service, a frequency of $8.82 \%$.

2) The epidemiological profile:

a) Maternity age: The age group of 15 - 19 years corresponding to teenage girls, was the most concerned, or $32.8 \%$. The average age of our patients was 24 years old with extremes of 15 to 43 years and a standard deviation of 7 years.

b) Parity: In our series, the nulliparas were the most numerous with $56 \%$.

c) Mode of admission: The patients coming from their home were the most concerned, i.e. $69.2 \%$.

d) Prenatal consultation: The patients who did not perform any ANC were the most numerous, i.e. $52 \%$.

e) Level of education: In our study, $68 \%$ of our patients were out of school.

f) Socioprofessionaldeliverry: The housewives were the most numerous, $44 \%$.

g) Risk factors for eclampsia: Primigestity was the main risk factor encountered, namely 52.4 .

3) Clinical aspect:

a) Gestational age: In our study, patients whose gestational age was greater than or equal to 37 SA were the most affected, or $62 \%$.

b) Reasons for consultation: Our results show that vertigo headache was the most frequent reason for consultation, with $76 \%$ and $68 \%$ respectively.

c) Type of hypertension and period of discovery: During our study, 120 cases of pre-eclampsia were obtained, i.e. $48 \%$; 70 cases of transient hypertension, or $28 \%$; 40 cases of pre-eclampsia added, $16 \%$; and 20 cases of chronic hypertension, or $8 \%$.

d) Clinical forms at admission: The most frequent clinical forms at admission were pre-eclampsia with $47.2 \%$, followed by eclampsia $23.2 \%$; and HRP $12 \%$. 


\section{4) Prognostic aspect:}

\section{a) Fetal prognosis:}

- APGAR score: In our series; $35.68 \%$ of newborn babies had an APGAR score $<7$ vs. $64.31 \%$ for an APGAR score $\geq 7$ at the 1 st minute. At the 5th minute, 25.5\% had an APGAR score $<7$ against $74.5 \%$ for an APGAR score $\geq 7$.

- Fetal Morbidity: In our series $83.52 \%$ of children born to hypertensive patients presented complications among which we have: fetal hypotrophy $(30.19 \%)$ and prematurity (23.92\%).

- Fetal lethality: In our study, we recorded 30 cases of MFIU and 7 cases of neonatal death out of 255 births, i.e. $14.50 \%$. Of the 7 cases of neonatal death, 3 occurred early between D0 and D7 and 4 were late from D7 to D28.

b) Fetal prognosis as a function of maternal uricemia: Fetal complications (hypotrophy, prematurity, SFA, and MFIU) were strongly correlated with maternal hyper-uricemia. In $90.90 \%$ of hypotrophy, $85.24 \%$ of premature infants, $95.55 \%$ of SFA, and $80 \%$ of MIU, the serum uric acid was greater than 350 mmol, while $85.71 \%$ of normal newborn infants were mothers. with normal serum.

c) Fetal prognosis according to maternal proteinuria: We recorded 204 children born to mothers with proteinuria greater than or equal to $30 \mathrm{mg} / \mathrm{dl}$, i.e. $80 \%$ of children. It should be noted that 6 children were of normal weight or $2.96 \%$.

\section{Discussion}

1) Frequency: During our study period, we recorded 250 hypertensive women out of a total of 2833 women who gave birth in the service, a frequency of $8.82 \%$. This result is superimposable to that reported by Kougang N.E. Conakry (Guinea) is $8.87 \%$ [14] and lower than that found by Kamano S.P. [15] to Kissidougou (Guinea) $11.14 \%$ in Guinea. On the other hand, it is higher than that found by Dao S.Z. [16] in Mali 3.65\%.

The high frequency in our study could be explained by the association of certain risk factors in the same patient such as multiparity, maternal age $<20$ years, but also by the fact that the maternity of Donka is a reference center for peripheral health centers and birthing centers in the city of Conakry. In addition, this is the capacity of the service (it is the largest motherhood in the country).

2) The epidemiological profile:

a) Maternity age: The age group of $15-19$ years corresponding to teenage girls, was the most concerned, or $32.8 \%$. The average age of our patients was 24 years old with extremes of 15 to 43 years and a standard deviation of 7 years.

This result is similar to those reported by Kougang N.E. [14], Kamano S.P. [15] and Sakouvogui B. [16] in the age group of 15 - 19 years respectively 35.6\%, $36.02 \%$ and $37.66 \%$. The high frequency of this age group could be justified by early marriages and the precocity of sexual intercourse.

b) Parity: In our series, the nulliparas were the most numerous with $56 \%$. This result is similar to those reported in the African literature [14] and this 
same literature reports that the relationship between hypertension and nulliparity can be explained by the maladjustment of the maternal organism to the upheavals of pregnancy when she is a young nulliparous, renal and placental haemodynamic disorders and uterine infantilism [17].

In addition, it seems that the black race is a risk factor in primiparous and not multiparous with a relative risk of 12 compared to the white race for reasons not yet elucidated [11].

c) Mode of admission: The patients coming from their home were the most concerned, i.e. $69.2 \%$. This result is close to the one found by Kougang N.E. in Guinea is $63.55 \%$ [14]. The high frequency in this series could be due to the poverty and ignorance of the latter because they note the changes taking place on their body; but for financial reasons or not knowing the evolution of these signs stay at home and arrive in our centers only when the sickness gets complicated.

d) Prenatal consultation: The patients who did not perform any ANC were the most numerous, i.e. $52 \%$. This result is superimposable to those found by Sakouvogui B. in Guinea [11] and Ngunga N. in Democratic Congo [18] i.e. $51.65 \%, 53.33 \%$ and $57.9 \%$ respectively.

The right CPN does not protect the woman from hypertension, but can detect in time and prevent complications, reason for which nowadays, we practice the refocused CPN which is to detect and treat pathologies encountered. It should be noted that the last CPN is the ultimate opportunity to identify risk factors and initiate the necessary measures for a delivery in the right conditions.

e) Level of education: In our study, $68 \%$ of our patients were out of school. This result is similar to those found by Koukang N.E. [14] and Kamano S.P. [15] respectively $64.8 \%$ and $65.12 \%$. The predominance of the out-of-school population could be explained by the fact that they are the most numerous in the general study population [14] and that illiterates have less information on reproductive health, thus exposing them to a lack or poor prenatal care that promotes the occurrence of hypertension during pregnancy and its complications.

f) Socioprofessional delivery: The housewives were the most numerous, $44 \%$. Kounkang N.E. [14] reports in his study a predominance among housewives is $39 \%$. For Beaufils M. [1], it seems that a low socio-economic level favors the installation of the HTA gravidic through an insufficient and unbalanced diet.

g) Risk factors for eclampsia: Primigestity was the main risk factor encountered, namely 52.4. This result is comparable to that found by Bah A.O. et al. who reported that $49.55 \%$ of their patients were Primigestes [19], and that of Vangeederhuysen et al. in NIGER who found $44.6 \%$ [8] During our study, the multiplicity of risk factors with 3 to 4 in the same patient was a very remarkable fact.

3) Clinical aspect:

a) Gestational age: In our study, patients whose gestational age was greater than or equal to $37 \mathrm{SA}$ were the most affected, or $62 \%$.

This result is greater than that of Kougang N.E. or $55.08 \%$ in patients whose gestational age was greater than or equal to $37 \mathrm{WA}$ [14]. The high frequency of 
hypertension at this level could be explained by the poor quality of CPN that does not detect high BP or significant proteinuria. In addition, the stress of childbirth can be the source of transient hypertension that increases the frequency of hypertension during pregnancy.

b) Reasons for consultation: Our results show that headache and vertigo were the most frequent reasons for consultation, with $76 \%$ and $68 \%$ respectively. These results are similar to those of Kougang N.E., $73.72 \%$. of her patients had consulted for headaches and $64.4 \%$ for vertigo [14].

c) Type of hypertension and period of discovery: During our study, 120 cases of pre-eclampsia were obtained, i.e. $48 \%$; 70 cases of transient hypertension, or 28\%; 40 cases of pre-eclampsia added, 16\%; and 20 cases of chronic hypertension, or $8 \%$. These results are similar to those of Kamano S.P. who obtained $48.84 \%$ pre-eclampsia, $16.28 \%$ added hypertension, and $20 \%$ transient hypertension; Against $24.17 \%$ of chronic hypertension [15]. Thiam M. et al. found $47 \%$ pre-eclampsia, $19 \%$ additional hypertension, $17 \%$ chronic hypertension, $17 \%$ transient hypertension [12].

\section{4) Prognostic aspect:}

a) Fetal prognosis:

APGAR score: In our series; $35.68 \%$ of newborns had an APGAR score $<7$ vs. $64.31 \%$ for an APGAR score $\geq 7$ at the 1 st minute. At the 5th minute, 25.5\% had an APGAR score $<7$ against 74.5\% for an APGAR score $\geq 7$.

Fetal Morbidity: In our series $83.52 \%$ of children born to hypertensive patients presented complications among which we have: fetal hypotrophy $30.19 \%$ and prematurity $23.92 \%$. This result on fetal hypotrophy is similar to those reported by Kougang N.E. [14] and Diallo M.H. [18], respectively $27.97 \%$ and $27.37 \%$, and higher than the literature data that $7 \%-20 \%$ of pregnancies with hypertension are complicated by fetal hypotrophy [19].

Fetal lethality: In our study, we recorded 30 cases of MFIU and 7 cases of neonatal death out of 255 births, i.e. $14.50 \%$. This result is lower than those reported by Kougang N.E. [14]; from Kamano S.P. [15]; Thiam M. et al. in Dakar [12] who found respectively $22.03 \% ; 24.44 \%$ and $50 \%$. Of the 7 cases of neonatal death, 3 occurred early between D0 and D7 and 4 were late from D7 to D28.

These children to varying degrees have been exposed to immediate complications: hypoglycaemia, hypothermia, infection with low resistance to microbial aggression accompanied most often by the difficulty of feeding.

b) Fetal prognosis as a function of maternal uricemia: Fetal complications (hypotrophy, prematurity, SFA, and MFIU) were strongly correlated with maternal hyper-uricemia. In $90.90 \%$ of hypotrophy, $85.24 \%$ of premature infants, $95.55 \%$ of SFA, and $80 \%$ of MIU, the serum uric acid was greater than 350 mmol, while $85.71 \%$ of normal newborn infants were mothers with normal serum. Hyperuric acid greater than $350 \mathrm{mmol} / \mathrm{l}$ influences fetal prognosis; she testifies hypo-volemia; it is correlated with the gravity of the volemic imbalances 
with the state of the child [5]. Urica remains the most accurate predictor of IUGR, chronic fetal distress, HRP, and Fetal Death in Utero [1].

c) Fetal prognosis according to maternal proteinuria: We recorded 204 children born to mothers with proteinuria greater than or equal to $30 \mathrm{mg} / \mathrm{dl}$, i.e. $80 \%$ of children. It should be noted that 6 children were of normal weight or $2.96 \%$. Fetal complications affected $97.04 \%$ of newborns: 60 children, or $29.41 \%$ by hypotrophy, 56 children or $27.45 \%$ by prematurity, 45 children or $22.05 \%$ by SFA, and $18,13 \%$ by the MFIU.

For Kougang N.E., $76.92 \%$ of her children were born with a mother with a proteinuria greater than or equal to $30 \mathrm{mg} / \mathrm{dl}$ [12]. Proteinuria is one of the defining elements of the severity of HTAG; once the hypertension is severe, it has fetal repercussions which could explain the complications observed; a correlation between the importance of proteinuria and an increase in perinatal mortality or IUGR has been demonstrated [5]. In fact, proteinuria testifies the existence of vascular glomerular lesions, particularly endotheliosis [1].

\section{Conclusion}

The detection of risk factors by a good prenatal follow-up and regular training of care providers for adequate and multidisciplinary care (obstetrician, nephrologist and pediatrician) of hypertensive pregnant women and their newborns can improve the maternal and fetal prognosis.

\section{Conflicts of Interest}

The authors declare no conflicts of interest regarding the publication of this paper.

\section{References}

[1] Beaufils, M. (2000) HTA of the Pregnant Woman. Annual of Internal Medicine, 4, 425-431.

[2] WHO (2005) Hypertensive Diseases of Pregnancy. World Health Report, 49.

[3] Dufour, F., Subtil, D. and Puesh, F. (2000) HTA and Pregnancy; Diagnosis, Complication, Treatment. Practitioner Review, 50, 1231-1237.

[4] Coomarasamy, A., Homest, H. Paoaioanou, S., et al. (2003) Aspirin for Prevention of Preeclampsia in Women with Bistorical Risk Factors: A Systematic Review. $O b$ stetrics \& Gynecology, 101, 1319-1332. https://doi.org/10.1016/S0029-7844(03)00169-8

[5] Sibony, O. (2004) HTA and Pregnancy; Biological and Clinical Aspects. Biology Slips, 51-55.

[6] Rasolonjatovo, J.D.C., Raherizaka, N., Rakotoarimanana, S., et al. (2005) Epidemiological and Clinical Study of Gravid Arterial Hypertension in Antsirabe [Madagascar]. Med. from Black Africa, 121-125.

[7] Lansac, J., Beger, C. and Magnin, G. (2000) HTA and Pregnancy. Obstetrics for the Practitioner. 3rd Edition, 165-175.

[8] Vangeenderhuysen, C.H., Banos, J.P. and Amadou, I.A. (2000) Hypertension in the Third Trimester of Pregnancy. A Study of Risk Factors Easily Identifiable at the First Prenatal Consultation in Niger. French Review of Obstetrics Gynecology, 353-356. 
[9] Cisse, C.T., Diem, M.E., N'gabo, D., Mbaye, M., Diane, P. and Moreau, J.C. (2003) Therapeutic Indication and Prognosis of Eclampsia at the University Hospital of Dakar. Journal of Gynecology Obstetrics, Reproductive Biology, 33, 239-245.

[10] Thiam, M., Goumbala, M., Gning, S.B., Fall, P.D., Cellier, C. and Perret, J.L. (2003) Maternal and Fetal Prognosis of the Association HTA and Pregnancy in Sub-Saharan Africa [Senegal]. Journal of Gynecology Obstetrics and Reproductive Biology, 32, 35-38.

[11] Sakouvogui, B. (2003) Hypertension and Pregnancy; Epidemiological, Clinical, Therapeutic and Prognosis Aspects at the Kindia Regional Hospital. Doctoral Thesis in Medicine, 33-58.

[12] Kougang, N.E. (2008) Hypertension Pregnancy; Management and Prognosis Maternal and Fetal Maternity IgnaceDeen CHU Conakry. Doctoral Thesis in Medicine, $10-54$.

[13] Kamano, S.P. (2008) Hypertension during Pregnancy; Epidemiology and Prognosis at the Maternity Ward of Kissidougou Prefectural Hospital. Doctoral Thesis, 16/58.

[14] Dao, S.Z. (2008) Hypertension and Pregnancy in the Gynecological Obstetrics Department of Gabriel Touré Hospital. Doctoral Thesis in Medicine; about 120 Cases. Mali Medical, 34-43.

[15] Luttun, A. and Carmellet, P. (2003) Soluble VEGF Receptor Sflt1: The Elusive Pre-Eclampsia Factor Discovered. Journal of Clinical Investigation, 111, 600-602. https://doi.org/10.1172/JCI18015

[16] Ngunga, N. (2003) Maternal and Fetal Prognosis during Severe Pre-Eclampsia. Medical Thesis, Simon Kimbangu University, Kinshasa, 49-64.

[17] Bah, O.A., Diallo, M.H., Conde, A.M. and Keita, N. (2001) Arterial Hypertension and Pregnancy; Maternal and Perinatal Mortality. Medicine of Black Africa, 461-464.

[18] Diallo, M.H. (2000) HTA and Pregnancy; Epidemiological, Clinical, and Evolving Aspects at the University Clinic of Gynecology and Obstetrics at Donka University Hospital. Doctoral Thesis in Medicine, 45-60.

[19] Provided, A. (2002) Major Complications of Pregnancy; HTA Pregnancy, Pre-Eclamptic Syndrome. Practitioner's Review, 52, 1345-1352. 